\title{
Extracting Hidden Symmetry from the Energy Spectrum
}

\author{
Emil A. Yuzbashyan ${ }^{1}$, William Happer ${ }^{1}$, Boris L. Altshuler ${ }^{1,2}$, Sriram B. Shastry ${ }^{3}$ \\ ${ }^{1}$ Physics Department, Princeton University, Princeton, NJ 08544 \\ ${ }^{2}$ NEC Research Institute, 4 Independence Way, Princeton, NJ 08540 \\ 3 Physics Department, Indian Institute of Science, Bangalore 560012, India
}

\begin{abstract}
In this paper we revisit the problem of finding hidden symmetries in quantum mechanical systems. Our interest in this problem was renewed by nontrivial degeneracies of a simple spin Hamiltonian used to model spin relaxation in alkali-metal vapors. We consider this spin Hamiltonian in detail and use this example to outline a general approach to finding symmetries when eigenvalues and eigenstates of the Hamiltonian are known. We extract all nontrivial symmetries responsible for the degeneracy and show that the symmetry group of the Hamiltonian is $S U(2)$. The symmetry operators have a simple meaning which becomes transparent in the limit of large spin. As an additional example we apply the method to the Hydrogen atom.
\end{abstract}

\section{Introduction}

The close connection between symmetry and degeneracy has been explored since the very foundation of Quantum Mechanics. Famous examples include degeneracies of the energy spectra in angular momentum in the 3d harmonic oscillator [1] and in the Hydrogen atom (well known as the accidental degeneracy) [2]. Here we undertake a detailed study of the connection between symmetries and degeneracies of a Hamiltonian that describes the exchange interaction of two spins, $\hat{\mathbf{S}}$ and $\hat{\mathbf{K}}$, and also includes Zeeman splitting for spin $\hat{\mathbf{S}}$ :

$$
\hat{H}(x)=x(K+1 / 2) \hat{S}_{z}+\hat{\mathbf{K}} \cdot \hat{\mathbf{S}}
$$

where $S=1$ and $K$ is arbitrary.

Our interest in this system was motivated by experiments [3] on spin relaxation in polarized alkalimetal vapors. In this case $\hat{\mathbf{S}}$ has a meaning of the total electronic spin, $\hat{\mathbf{K}}$ is the nuclear spin, and the dimensionless constant $x$ represents the magnetic field. A diagram of energy levels of Hamiltonian (1) for a typical value of $K=2$ is shown on Fig. 1. In addition to degeneracies at $x=0$ (no Zeeman splitting) the spectrum displays less trivial $(2 K+1)$-fold degeneracies at $x= \pm 1$. These degeneracies show up as resonances in the spin relaxation rate and provide the key evidence for a particular mechanism of spin relaxation (see [3] for details). The degeneracies at $x= \pm 1$ have been also discussed in [4].

Despite the simplicity of Hamiltonian (1), it is not trivial to determine its symmetry at $x=1$. To the best of our knowledge there is no general textbook algorithm for extracting symmetries. In this 
paper we propose such an algorithm. We demonstrate how, using only a minimal intuition into the reasons for degeneracy, one can find hidden symmetries whenever the eigenstates and the eigenvalues of the Hamiltonian are known.

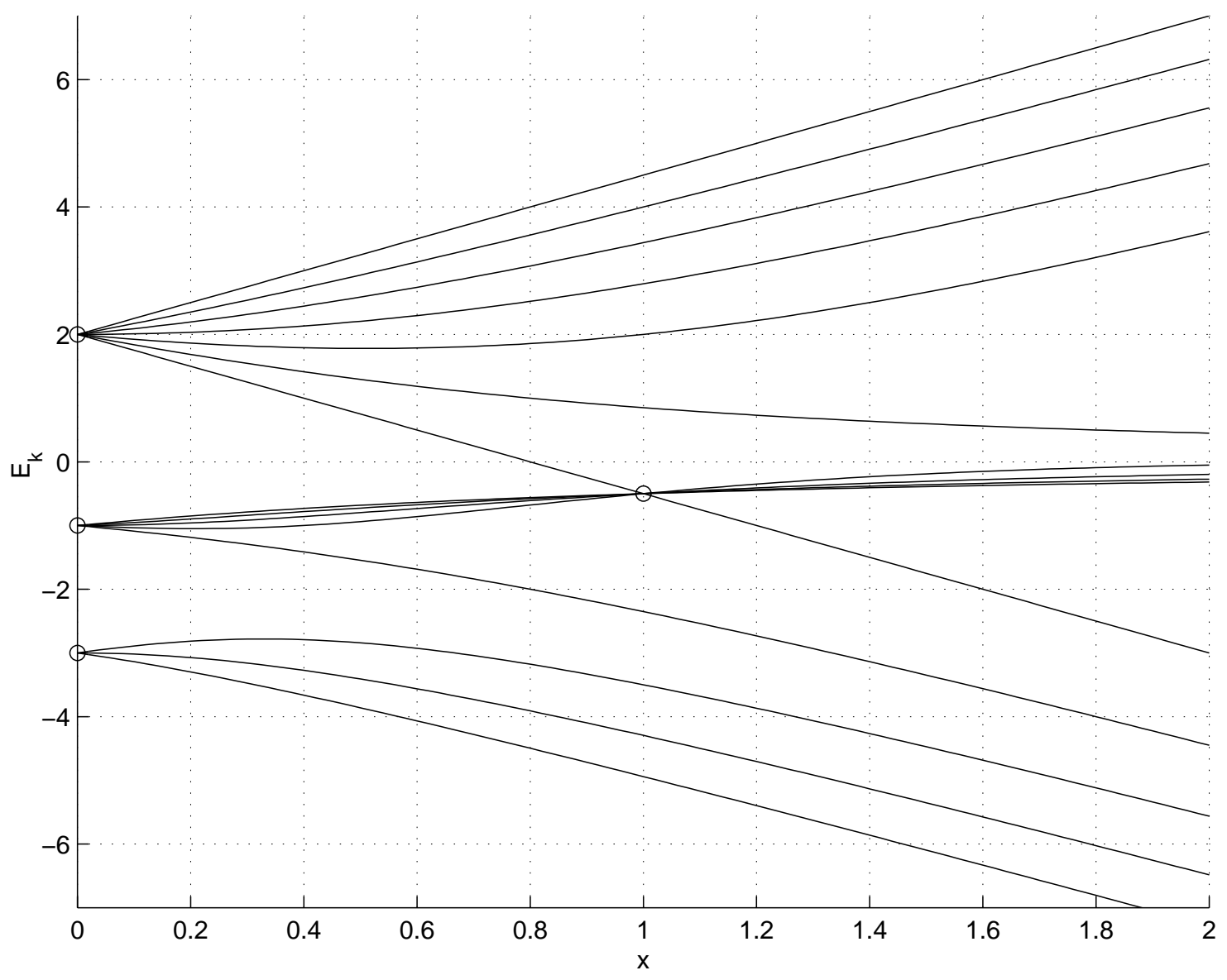

Figure 1: Eigenvalues of $\hat{H}(x)=x(K+1 / 2) \hat{S}_{z}+\hat{\mathbf{K}} \cdot \hat{\mathbf{S}}$ for $S=1$ and $K=2$. Note the level crossings at $x=1$.

Before proceeding further let us formulate the general problem of identifying the symmetry responsible for a particular spectral degeneracy. Symmetries manifest themselves through operators that commute with the Hamiltonian, $\hat{H}$. Consider a set of these operators $\mathcal{A}=\left\{\hat{A}_{i}\right\}$. If not all of the operators $\hat{A}_{i}$ commute with each other, this symmetry implies some spectral degeneracy. Indeed, if

$$
\left[\hat{A}_{i}, \hat{H}\right]=\left[\hat{A}_{j}, \hat{H}\right]=0 \quad \text { and } \quad\left[\hat{A}_{i}, \hat{A}_{j}\right] \neq 0,
$$

there exists an eigenstate of the Hamiltonian $|\alpha\rangle$ for which $\hat{A}_{i}|\alpha\rangle \neq \hat{A}_{j}|\alpha\rangle$. This state is necessarily degenerate, since according to equation (2) $\hat{A}_{i}|\alpha\rangle$ and $\hat{A}_{j}|\alpha\rangle$ are eigenstates of $\hat{H}$ of the same energy as $|\alpha\rangle$ (see e.g. [1]). 
Is a particular set of operators $\mathcal{A}$ sufficient to account for given spectral degeneracies? The positive answer to this question implies that

1. all degenerate states can be labelled by eigenvalues of a number of mutually commuting operators from the set $\mathcal{A}$

2. any degenerate state can be obtained from any other state in the same eigenspace by a repeated action of operators $\hat{A}_{i}$ or their linear combinations.

Provided that conditions 1) and 2) are met one can complete the analysis by clarifying the physical significance of operators $\left\{\hat{A}_{i}\right\}$. An additional, more abstract, question one might ask is what is the group generated by operators $\left\{\hat{A}_{i}\right\}$.

Consider for example the degeneracy of Hamiltonian (1) at $x=0$. One can introduce $\hat{\mathbf{G}}=\hat{\mathbf{K}}+\hat{\mathbf{S}}$ and identify, e.g., $\hat{A}_{1}=\hat{G}_{x}$ and $\hat{A}_{2}=\hat{G}_{y}$. The commutator of $\hat{A}_{1}$ with $\hat{A}_{2}$ yields $\hat{A}_{3}=\hat{G}_{z}$. Eigenvalues of $\hat{G}_{z}$ and $\hat{\mathbf{G}}^{2}$ can be used to label the degenerate states, while linear combinations of $\hat{G}_{x}$ and $\hat{G}_{y}$, $\hat{G}_{+}$and $\hat{G}_{-}$, connect all degenerate states in a given eigenspace. Finally, any operator that commutes with $\hat{H}(0)$ can be written in terms of $\hat{G}_{x}, \hat{G}_{y}, \hat{G}_{z}$, and $\hat{\mathbf{G}}^{2}$. The group generated by the components of $\hat{\mathbf{G}}$ is $S U(2)$ and the group elements have a meaning of rotations in 3 d space.

Frequently, we encounter a situation when the spectrum is known exactly and yet the symmetries are hidden. In such cases the following formal expression for commuting operators serves as a useful starting point. One can show quite generally that, up to an operator that annihilates all degenerate states, all integrals of motion must be of the form

$$
\hat{X}=\sum_{n} \hat{P}_{n} \hat{Y} \hat{P}_{n}
$$

Here $\hat{P}_{n}$ is an operator that projects out the $n$th degenerate eigenspace and $\hat{Y}$ is an arbitrary operator. We prove equation (3) in the Appendix. The problem of finding the symmetry thus reduces to making appropriate choices for $\hat{Y}$. This choice can be either guided by limiting cases, e.g., the classical limit, where the symmetry is simple to identify, or by an intuition as to what type of conservation laws (e.g. scalars or vectors or etc.) one expects to find. However, examples we considered show that many apparently different choices for the operator $\hat{Y}$ produce equivalent conservation laws. Thus, it is usually sufficient to explore the simplest options - the basic operators of the problem. For example, if the Hamiltonian is written in terms of $\hat{\mathbf{r}}$ and $\hat{\mathbf{p}}$, natural choices for $\hat{Y}$ would be $\hat{\mathbf{r}}^{2}$ and $\hat{\mathbf{p}}^{2}$ if one is looking for a conserved scalar or $\hat{\mathbf{r}}$ and $\hat{\mathbf{p}}$ if a conserved vector is expected.

The paper is organized as follows. First, we determine and analyze in detail the spectrum of Hamiltonian (1) at $x=1$ (Section 2). We show that, apart from the zero field degeneracy at $x=0$, there are degeneracies only at $x= \pm 1$, the spectrum at $x=-1$ being simply related to that at $x=1$. Given the eigenstates, integrals of motion (3) can be evaluated without knowing projectors $\hat{P}_{n}$ explicitly. In this case the general strategy is to compute the matrix elements of $\hat{X}$ in equation (3) and use them to determine $\hat{X}$ in terms of basic operators of the problem. We will illustrate this approach in Section 6 . 
In Section 3 we use (3) to explicitly derive commuting operators that connect all degenerate states at $x=1$. We demonstrate that the symmetry group of Hamiltonian (1) at $x=1$ is $S U(2)$. The physical meaning of symmetry operators is clarified in Section 4, where we consider the limit of large $K$.

In Section 5 we rewrite the Hamiltonian in the form that makes the analogy to the large $K$ limit particularly clear and allows us to establish some additional properties of the energy spectrum. In Section 6 we provide another example of the general approach of finding symmetries based on (3) by deriving the Runge-Lenz vector from (3).

\section{Energy Spectrum}

Here we analyze the spectrum of Hamiltonian (1) at arbitrary $x$ and consider in detail the spectrum at $x=1$. The results discussed in this section were originally derived in [6]. We will follow [6] closely adopting in most cases the same notation.

First, we note that the $z$ component of the total spin is conserved.

$$
\left[\hat{G}_{z}, \hat{H}(x)\right]=0
$$

Since $S=1$, there are at most three independent states for each eigenvalue $m$ of $\hat{G}_{z}$. In the basis $\left|K_{z}, S_{z}\right\rangle$ these states are $|m-1,1\rangle,|m, 0\rangle$, and $|m+1,-1\rangle$. The block of the Hamiltonian (1) that corresponds to $m$ for $|m| \leq K-1$ is

$$
\hat{H}=\frac{1}{\sqrt{2}}\left[\begin{array}{ccc}
\sqrt{2}(x(K+1 / 2)+m-1) & \sqrt{(K+m)(K-m+1)} & 0 \\
\sqrt{(K+m)(K-m+1)} & 0 & \sqrt{(K-m)(K+m+1)} \\
0 & \sqrt{(K-m)(K+m+1)} & \sqrt{2}(-x(K+1 / 2)-m-1)
\end{array}\right]
$$

with the eigenvalue equation

$$
\begin{aligned}
& E^{3}+2 E^{2}-a(x) E-K^{2}-b(x)=0 \\
& a(x)=\left(1+x^{2}\right)(K+1 / 2)^{2}+2 x(K+1 / 2) m-5 / 4 \\
& b(x)=K^{2}+K+x(K+1 / 2) m
\end{aligned}
$$

In addition to $3 \times 3$ blocks (5) the Hamiltonian also has two $2 \times 2$ blocks for $|m|=K$ and two $1 \times 1$ blocks for $|m|=K+1$. Energies for these values of $m$ are also solutions of (6).

Degeneracies occur only at $x=0$ and $x= \pm 1$ (see Fig. 1). The energy spectrum at $x=-1$ is identical to that at $x=1$ while the eigenstates are related via a unitary transformation (a rotation by $\pi$ around any axis in $x y$ plane). Therefore, it is sufficient to consider only $x=1$.

The spectrum at $x=1$ has the following features: 
- There are $2 K+1$ degenerate states $|D m\rangle$ ("D" for degenerate states) with the energies $E_{D m}=-1 / 2$

- There is a gap at $m=-K$ in the values of $m$ that can be assigned to degenerate states $|D m\rangle$, $m=K, K-1, \ldots,-K+1$ and $-K-1$

- There are $2 K+2$ non-degenerate states $|T m\rangle$ ("T" for top states) with energies $E_{T m}>-1 / 2$ and $m=K+1, K, \ldots,-K$

- There are $2 K$ non-degenerate states $|B m\rangle$ ("B" for bottom states) with energies $E_{B m}<-1 / 2$ and $m=K-1, K, \ldots,-K$

- Energies of the top $|T m\rangle$ and bottom $|B m\rangle$ states are related by $E_{T m}+E_{B m}=-3 / 2(|m|<K)$

Finally, we can use (5) to compute the energies at $x=1$ and the wave-functions $|D m\rangle$ of the degenerate states

$$
\begin{gathered}
E_{D m}=-1 / 2 \quad E_{T m}=-3 / 4+\sqrt{\mathrm{B}(m)^{2}+1 / 16} \quad E_{B m}=-3 / 2-E_{T m} \\
{\left[\begin{array}{l}
\langle m-1,1 \mid D m\rangle \\
\langle m, 0 \mid D m\rangle \\
\langle m+1,-1 \mid D m\rangle
\end{array}\right]=\frac{1}{\sqrt{2} \mathrm{~B}(m)}\left[\begin{array}{c}
-\sqrt{(K+1+m)(K+1-m)} \\
\sqrt{2(K+1+m)(K+m)} \\
\sqrt{(K+m)(K-m)}
\end{array}\right]}
\end{gathered}
$$

where

$$
\mathrm{B}(m)=(2 K+1)(K+m+1 / 2)
$$

\section{Symmetries}

In this section we derive integrals of motion responsible for degeneracies at $x=1$. We write them in terms of components of $\hat{\mathbf{S}}$ and $\hat{\mathbf{K}}$ and show that the symmetry group is $S U(2)$.

Our starting point is the general expression for commuting operators (3). Hamiltonian (1) has only one degenerate subspace at $x=1$, so there is only one projection operator in (3).

$$
\hat{X}=\hat{P} \hat{Y} \hat{P}
$$

Even though we do not need to know projection operators explicitly to evaluate (3), in the case of Hamiltonian (1) the projector $\hat{P}$ has a simple meaning in the large $K$ limit, so we derive it bellow from the eigenvalue equation (6).

When the energy $E$ is replaced by $\hat{H}$ and $m$ is replaced by $\hat{G}_{z}$, eigenvalue equation (6) translates into a cubic identity for the Hamiltonian [6]. At $x=1$ this identity can be factored out as follows

$$
(\hat{H}+1 / 2) \underbrace{\left[\hat{\mathrm{B}}\left(\hat{G}_{z}\right)^{2}-(\hat{H}+1 / 2)(\hat{H}+1)\right]}_{\hat{\Pi}}=0
$$


where

$$
\hat{\mathrm{B}}\left(\hat{G}_{z}\right)=(2 K+1)\left(K+\hat{G}_{z}+1 / 2\right)
$$

States with $E=-1 / 2$ are degenerate with respect to the eigenvalues of $\hat{G}_{z}$. The operator in the square brackets, $\hat{\Pi}$, is then an "unnormalized projection operator" that projects out the degenerate subspace.

$$
\hat{\Pi}|N m\rangle=0 \quad \hat{\Pi}|D m\rangle=\mathrm{B}(m)^{2}|D m\rangle
$$

The operator $\hat{\Pi}$ can be normalized to a usual projection operator for the degenerate subspace [6].

$$
\hat{P}=\frac{\hat{\Pi}}{\hat{\mathrm{B}}\left(\hat{G}_{z}\right)^{2}}=1-\frac{(\hat{H}+1 / 2)(\hat{H}+1)}{\hat{\mathrm{B}}\left(\hat{G}_{z}\right)^{2}}
$$

Now we have to identify appropriate choices for the operator $\hat{Y}$ in equation (10). Let us first try the simplest options - operators that are linear in components of $\hat{\mathbf{S}}$ and $\hat{\mathbf{K}}$. Consider, for example, a conserved vector ${ }^{1}$

$$
\hat{\mathbf{L}}=\hat{P} \hat{\mathbf{K}} \hat{P}
$$

Let us determine the action of components of $\hat{\mathbf{L}}$ on the eigenstates of Hamiltonian. Since $\hat{P}$ is a projector for the degenerate subspace ${ }^{2}$,

$$
\hat{L}_{i}|N m\rangle=0 \quad i=1,2,3 .
$$

To calculate the action of $\hat{L}_{i}$ on degenerate states recall that due to selection rules (see e.g. [1]) the components of a vector can have nonzero matrix elements only for transitions $m \rightarrow m$ and $m \rightarrow m \pm 1$. We note also the following relation between matrix elements of operators $\hat{X}$ and $\hat{Y}$ in equation (10)

$$
\left\langle m^{\prime}|\hat{X}| m\right\rangle=\left\langle D m^{\prime}|\hat{Y}| D m\right\rangle
$$

where $|m\rangle$ and $\left|m^{\prime}\right\rangle$ are any two eigenstates of $\hat{G}_{z}$ with eigenvalues $m$ and $m^{\prime}$ respectively. Using equations (16) and (8), we obtain

$$
\hat{L}_{+}|D m\rangle=\sqrt{\frac{\left(K^{2}-m^{2}\right)\left((2 K+2 m+3)^{2}-1\right)}{(2 K+2 m+2)^{2}-1}}|D, m+1\rangle
$$

The action of $\hat{L}_{-}$can be determined directly from equation (17) and the action of $\hat{L}_{z}$ from equations (16) and (8) with $m^{\prime}=m$.

Note that equation (17) is not well defined for $m=-K-1$. For the state $|D,-K-1\rangle$ instead of equation (17) we get

$$
\hat{L}_{ \pm}|D,-K-1\rangle=0
$$

\footnotetext{
${ }^{1}$ Projection operator $\hat{P}$ is a scalar, because the Hamiltonian is a scalar. To see this the operator $\hat{S}_{z}$ in Hamiltonian (1) should be written as $\hat{\mathbf{S}} \cdot \mathbf{b}$, where $\mathbf{b}$ is a unit vector along the magnetic field. By the same argument $\hat{G}_{z}=\hat{\mathbf{G}} \cdot \mathbf{b}$ is a conserved scalar. Therefore, $\mathbf{L}$ is a vector.

${ }^{2}$ We use the usual notation $\hat{L}_{1} \equiv \hat{L}_{x} \equiv \frac{\hat{L}_{+}+\hat{L}_{-}}{2}, \hat{L}_{2} \equiv \hat{L}_{y} \equiv \frac{\hat{L}_{+}-\hat{L}_{-}}{2 i}$, and $\hat{L}_{3} \equiv \hat{L}_{z}$.
} 
Equation (18) reflects the existence of a gap at $m=-K$ in the eigenvalues of $\hat{G}_{z}$ assigned to degenerate states. An operator that connects the state $|D,-K-1\rangle$ to the rest of degenerate subspace must change $m$ by at least two. According to selection rules such an operator is neither a scalar nor a component of a vector. Let us first consider values of $m \neq-K-1$ and revisit the problem of the gap at the end of the section.

Since equation (17) is not of the standard form for a raising operator in $s u(2)$, commutation relations for $\hat{L}_{ \pm}$are "deformed" versions of $s u(2)$ commutation relations. However, one can derive usual $s u(2)$ operators from $\hat{L}_{ \pm}$and $\hat{G}_{z}$. Define

$$
\hat{N}_{+}=\left(\hat{N}_{-}\right)^{\dagger}=\hat{A}\left(\hat{G}_{z}\right) \hat{A}\left(\hat{G}_{z}+1\right) \hat{L}_{+} \quad \hat{N}_{z}=\left(\hat{G}_{z}-1 / 2\right) \hat{P}
$$

where

$$
\hat{A}\left(\hat{G}_{z}\right)=\frac{\hat{\mathrm{B}}\left(\hat{G}_{z}\right)}{\hat{\mathrm{B}}\left(\hat{G}_{z}+1 / 2\right)}
$$

Operators $\hat{N}_{i}$ form an $s u(2)$ algebra:

$$
\left[\hat{N}_{i}, \hat{N}_{j}\right]=\varepsilon_{i j k} \hat{N}_{k}
$$

All degenerate states with $m \neq-K-1$ are connected by operators $N_{ \pm}$. Each of these states is labelled by $N_{z}$ and by an eigenvalue of the Casimir operator

$$
\hat{\mathbf{N}}^{2} \equiv \sum_{i=1}^{3} \hat{N}_{i}^{2}=\hat{N}_{-} \hat{N}_{+}+\hat{N}_{z}^{2}+\hat{N}_{z}
$$

Operator $\hat{\mathbf{N}}^{2}$ has eigenvalues $N(N+1)$ with (half)integer values of $N$.

$$
|D m\rangle=\left|N=K-1 / 2, N_{z}=m-1 / 2\right\rangle \quad m \neq-K-1
$$

Thus, degenerate states with $m \neq-K-1$ transform under the $2 K$-dimensional representation of this $s u(2)$, while nondegenerate states are singlets.

Note that by selection rules taking any other vector, e.g. a linear combination of $\hat{\mathbf{K}}$ and $\hat{\mathbf{S}}$, instead of $\hat{\mathbf{K}}$ in the definition of $\hat{\mathbf{L}}$, equation (15), will produce the same results and lead to the same $s u(2)$ algebra.

As was mentioned above, to connect the state $|D,-K-1\rangle$ to the rest of the degenerate subspace, one needs an operator that can change $m$ by at least two. Let us consider, for example, operators

$$
\hat{M}_{ \pm}=\hat{P} \hat{S}_{ \pm}^{2} \hat{P}
$$

Using operators $\hat{M}_{ \pm}$and operators $\hat{N}_{i}$, one can construct a new $s u(2)$ algebra with a representation that incorporates the state $|D,-K-1\rangle$. We define

$$
\begin{aligned}
& \hat{J}_{+}=\left(\hat{J}_{-}\right)^{\dagger}=\sqrt{\frac{K+\hat{G}_{z}}{K+\hat{G}_{z}-1}} \hat{N}_{+}-\sqrt{3 K+3 / 2} \hat{P} \hat{S}_{+}^{2} \hat{P}_{-K-1} \\
& \hat{J}_{z}=\hat{G}_{z} \hat{P}+\hat{P}_{-K-1}
\end{aligned}
$$


Here $^{3} \hat{P}_{-K-1}$ denotes the projector onto the state $|D,-K-1\rangle$

$$
\hat{P}_{-K-1}|D,-K-1\rangle=|D,-K-1\rangle \quad \hat{P}_{-K-1}|m \neq-K-1\rangle=0
$$

The operator $\hat{P}_{-K-1}$ can be written in terms of $\hat{G}_{z}$ as follows

$$
\hat{P}_{-K-1}=\frac{1}{(2 K+2) !} \prod_{m \neq-K-1}\left(m-\hat{G}_{z}\right)
$$

One can check by a direct computation using equations (21), (19), (17), and (14) that

1. Operators $\hat{J}_{ \pm}$and $\hat{J}_{z}$ commute with Hamiltonian (1) at $x=1$ and form an $s u(2)$ algebra.

2. All degenerated states are uniquely labelled by eigenvalues of $\hat{J}_{z}$ and $\hat{\mathbf{J}}^{2}$

$$
|D m\rangle=\left|J=K, J_{z}=m\right\rangle \quad-K+1 \leq m \leq K
$$

and

$$
|D,-K-1\rangle=\left|J=K, J_{z}=-K\right\rangle
$$

Thus, $J_{z}$ runs from $-K$ to $K$ with no gap and the degenerate subspace transforms under a $(2 \mathrm{~K}+1)$-dimensional representation of the $s u(2)$ defined by equations $(21)$.

3. All nondegenerate states are singlets, i.e. they have $J=J_{z}=0$

Note that operators $\hat{M}_{ \pm}$are linear combinations of components of a conserved rank 2 tensor $\hat{M}_{i j}=\hat{P} \hat{S}_{i} \hat{S}_{j} \hat{P}$. By the same argument as for vectors, inserting any other rank 2 tensor instead of operator $\hat{Y}$ in equation (10) will result in equivalent operators. Higher rank tensors are not needed. Indeed, operators $\hat{J}_{i}$ already connect all degenerate states. Therefore, components of tensors of a rank greater than two can be written in terms of products of operators $\hat{J}_{i}$ in the same way as components of any tensor that commutes with Hamiltonian (1) at $x=0$ can be expressed through $\hat{G}_{x}, \hat{G}_{y}$, and $\hat{G}_{z}$.

\section{Large $K$ Limit}

To gain some insight into the conservation laws derived in the previous section let us consider the limit $K \gg 1$. In this limit the meaning of symmetry turns out to be transparent. Our intention in this section is not to present a rigorous analysis, but rather to develop an intuition about symmetries responsible for degeneracy.

If $\hat{\mathbf{K}}$ is a classical vector, we can write the Hamiltonian at $x=1$ as

$$
\hat{H}_{c l}=|(K+1 / 2) \mathbf{z}+\hat{\mathbf{K}}| \hat{S}_{\mathbf{n}}
$$

\footnotetext{
${ }^{3}$ Note that $\hat{P}_{-K-1}=\hat{P}_{-K-1} \hat{P}=\hat{P} \hat{P}_{-K-1} \hat{P}$ and $\hat{G}_{z} \hat{P}=\hat{P} \hat{G}_{z}=\hat{P} \hat{G}_{z} \hat{P}$. Hence, operators $J_{ \pm}$and $J_{z}$ defined by equations (21) are indeed of the general form (3).
} 
where $\mathbf{z}$ is a unit vector along the $z$-axis and operator $\hat{S}_{\mathbf{n}}$ is the projection of $\hat{\mathbf{S}}$ onto the axis parallel to the vector $(K+1 / 2) \mathbf{z}+\hat{\mathbf{K}}$. For each orientation of $\hat{\mathbf{K}}$ there are three energies corresponding to $S_{\mathbf{n}}=+1$, -1 and 0 - "top", "bottom" and "middle" levels. Middle levels have zero energy, independent on the direction of $\hat{\mathbf{K}}$.

Now let us take into account quantization of $\hat{\mathbf{K}}$ in the limit $K \gg 1$. We assume nevertheless that $\hat{S}_{\mathbf{n}}$ still can be interpreted as a projection of $\hat{\mathbf{S}}^{4}$.

The direction of $\hat{\mathbf{K}}$ is specified by $K_{z} \approx m$. States $\left|S_{\mathbf{n}}=0, G_{z}=m\right\rangle$ are degenerate with respect to $m$. Evidently, symmetries responsible for this degeneracy "rotate $\hat{\mathbf{K}}$ " for states with $\hat{S}_{\mathbf{n}}=0$, while keeping it unchanged for states with $\hat{S}_{\mathbf{n}} \neq 0$. Generators for such rotations are

$$
\hat{L}_{x, y}^{c l}=\left(1-\hat{S}_{\mathbf{n}}^{2}\right) \hat{K}_{x, y}\left(1-\hat{S}_{\mathbf{n}}^{2}\right)
$$

Operators $\hat{L}_{x, y}^{c l}$ commute with the Hamiltonian (22) which follows from the spin-1 identity

$$
\hat{S}_{\mathbf{n}}\left(1-\hat{S}_{\mathbf{n}}^{2}\right)=0
$$

In deriving (23) we made use of a projection operator

$$
\hat{P}_{c l}=1-\hat{S}_{\mathbf{n}}^{2} \quad \hat{P}_{c l}|D m\rangle=|D m\rangle \quad \hat{P}_{c l}|N m\rangle=0
$$

where $|D m\rangle$ and $|N m\rangle$ denote degenerate and non-degenerate states respectively. Operators $\hat{L}_{x, y}^{c l}$ together with $\hat{L}_{z}^{c l}=\left(1-\hat{S}_{\mathbf{n}}^{2}\right) \hat{K}_{z}\left(1-\hat{S}_{\mathbf{n}}^{2}\right)$ form an $s u(2)$ algebra

$$
\left[\hat{L}_{i}^{c l}, \hat{L}_{j}^{c l}\right]=i \varepsilon_{i j k} \hat{L}_{k}^{c l}+O(1 / K)
$$

The degenerate subspace transforms under a $2 K+1$ dimensional representation of this $s u(2)$, while nondegenerate states are singlets. Neglecting terms of the order of $1 / K$, we can write Hamiltonian (22) as

$$
\hat{H}_{c l}=\hat{\mathrm{B}}\left(\hat{G}_{z}\right) \hat{S}_{\mathbf{n}}
$$

Note that the operator $\hat{\mathrm{B}}\left(\hat{G}_{z}\right)$ defined by equation (12) can be interpreted as an "effective magnetic field operator", which explains notation (12). Combining equation (27) with spin-1 identity (24), we obtain

$$
\begin{gathered}
\hat{S}_{\mathbf{n}}^{2}=\frac{\hat{H}_{c l}^{2}}{\hat{\mathrm{B}}\left(\hat{G}_{z}\right)^{2}} \quad \hat{P}_{c l}=1-\frac{\hat{H}_{c l}^{2}}{\hat{\mathrm{B}}\left(\hat{G}_{z}\right)^{2}} \\
\hat{H}_{c l}\left[\hat{\mathrm{B}}\left(\hat{G}_{z}\right)^{2}-\hat{H}_{c l}^{2}\right]=0
\end{gathered}
$$

Now let us compare these results to the "quantum" case. First, one can check that operators $\hat{L}_{i}^{c l}, \hat{H}_{c l}$, and $\hat{P}_{c l}$ defined above are indeed the limits of the corresponding operators introduced in the previous section. Namely,

$$
\hat{H} \rightarrow \hat{H}_{c l} \quad \hat{P} \rightarrow \hat{P}_{c l} \quad \hat{J}_{i} \rightarrow \hat{N}_{i} \rightarrow \hat{L}_{i} \rightarrow \hat{L}_{i}^{c l} \quad \text { as } K \rightarrow \infty
$$

\footnotetext{
${ }^{4}$ The large $K$ limit can be treated more accurately by introducing a preferred direction for $\hat{\mathbf{K}}$ and representing $\hat{\mathbf{K}}$ in terms of Holstein-Primakoff bosons.
} 
Therefore, we can think of the operator

$$
\hat{I}_{n}^{2} \equiv \frac{(\hat{H}+1 / 2)(\hat{H}+1)}{\hat{\mathrm{B}}\left(\hat{G}_{z}\right)^{2}},
$$

appearing in the definition of $\hat{P}$, equation (14), as a projection of a spin one onto a "quantum axis" along $(K+1 / 2) \mathbf{z}+\hat{\mathbf{K}}$. Further, the spin-1 identity, equation (24), and its descendent equation (29) are the limit of equation (11). Equation (30) shows that the difference between $\hat{N}_{i}$ and $\hat{J}_{i}$ disappears in the limit of large $K$. Thus, the gap in the values of $m$ has no analogs in the leading order of this limit. Note also that in the leading order in $1 / K$ the middle levels of Hamiltonian (1) are degenerate at any $x$ and for all integer values of $S$. An analysis of the subleading corrections to the large $K$ limit may provide a simple explanation of why the degeneracies occur only for $S=1$.

\section{$5 \quad$ Generalized Symmetries}

In this section we deviate somewhat from the main topic of the paper and discuss generalized symmetries of Hamiltonian (1). Generalized symmetries are anticommutation relations that explain, for example, the following relation between energies of top and bottom levels (see Section 2):

$$
E_{T m}+E_{B m}=-3 / 2 \quad|m|<K-1
$$

We also show that the analogy to the large $K$ limit can be pushed even further if we replace the Hamiltonian (1) at $x=1$ with an effective Hamiltonian by keeping only the blocks with $|m|<K-1$.

First, we note that there exists a basis where the block of the Hamiltonian (5) at $x=1$ reads

$$
\left[\begin{array}{ccc}
-1 & \mathrm{~B}(m) & 0 \\
\mathrm{~B}(m) & -1 / 2 & 0 \\
0 & 0 & -1 / 2
\end{array}\right]
$$

Indeed, matrix (32) has the same eigenvalue equation as matrix (5) at $x=1$. Therefore, matrices (32) and (5) represent the same operator in two different basises. From equation (31) we find that $\hat{I}_{\mathbf{n}}^{2}$ for $|m|<K$ in this basis is

$$
\hat{I}_{\mathbf{n}}^{2}=\left[\begin{array}{lll}
1 & 0 & 0 \\
0 & 1 & 0 \\
0 & 0 & 0
\end{array}\right]
$$

Comparing matrix (32) and equation (27), and using (33), we conclude that it is natural to define $\hat{I}_{\mathbf{n}}$ as

$$
\hat{I}_{\mathbf{n}}=\left[\begin{array}{lll}
0 & 1 & 0 \\
1 & 0 & 0 \\
0 & 0 & 0
\end{array}\right]
$$


Thus, we can write (cf. (27))

$$
\hat{H}_{e f f}=\hat{\mathrm{B}}\left(\hat{G}_{z}\right) \hat{I}_{1}-1 / 4 \hat{I}_{3}-1 / 4 \hat{I}_{1}^{2}-1 / 2
$$

where $\hat{I}_{1}, \hat{I}_{2}$, and $\hat{I}_{3}$ are the first three Gell-Mann matrices of $s u(3)$.

$$
\hat{I}_{1} \equiv \hat{I}_{\mathbf{n}}=\left[\begin{array}{lll}
0 & 1 & 0 \\
1 & 0 & 0 \\
0 & 0 & 0
\end{array}\right] \quad \hat{I}_{2}=\left[\begin{array}{ccc}
0 & -i & 0 \\
i & 0 & 0 \\
0 & 0 & 0
\end{array}\right] \quad \hat{I}_{3}=\left[\begin{array}{ccc}
1 & 0 & 0 \\
0 & -1 & 0 \\
0 & 0 & 0
\end{array}\right]
$$

Matrices $\hat{I}_{1}, \hat{I}_{2}$, and $\hat{I}_{3}$ generate an $s u(2)$ subgroup of $s u(3)$ known as the isospin subgroup. We note that

$$
\hat{H}_{e f f} \hat{I}_{2}+\hat{I}_{2} \hat{H}_{e f f}=-\frac{3}{2} \hat{I}_{2}
$$

Anticommutation relations of this type are sometimes called generalized symmetries. Equation (36) shows that if $|m\rangle$ is an eigenstate of the Hamiltonian at $x=1$ with $|m|<K$ and the energy $E, \hat{I}_{2}|m\rangle$ is either also an eigenstate but with the energy $-3 / 2-E$ or $\hat{I}_{2}|m\rangle=0$. Examining the eigenstates of matrix (32) one can verify that $\hat{I}_{2}|N m\rangle \neq 0$ and $\hat{I}_{2}|D m\rangle=0$. Therefore, we get

$$
E_{T m}+E_{B m}=-\frac{3}{2} \quad \hat{I}_{2}|T m\rangle=|B m\rangle \quad \hat{I}_{2}|B m\rangle=|T m\rangle \quad \hat{I}_{2}|D m\rangle=0
$$

Note also that since for each $|m|<K$ the operator $\hat{\mathbf{K}} \cdot \hat{\mathbf{S}}$ has three eigenvalues $-K-1,-1$, and $K$

$$
E_{T m}+E_{D m}+E_{B m}=\operatorname{Tr}(\hat{H})=(K+1 / 2) \operatorname{Tr}\left(\hat{S}_{z}\right)+\operatorname{Tr}(\hat{\mathbf{K}} \cdot \hat{\mathbf{S}})=-2
$$

\section{Hydrogen Atom}

Here we derive the nontrivial conservation laws for the Hydrogen atom from the general expression (3).

The Hamiltonian in atomic units (see e.g. [1]) is

$$
\hat{H}=\frac{\hat{\mathbf{p}}^{2}}{2}-\frac{1}{\hat{r}}
$$

In addition to the angular momentum $\hat{\mathbf{L}}=\hat{\mathbf{r}} \times \hat{\mathbf{p}}$, Hamiltonian (39) also conserves the Runge-Lenz vector

$$
\hat{\mathbf{A}}=\frac{\hat{\mathbf{r}}}{r}-\frac{1}{2}(\hat{\mathbf{p}} \times \hat{\mathbf{L}}-\hat{\mathbf{L}} \times \hat{\mathbf{p}})
$$

Integrals of motion (40) explain the degeneracy in angular momentum. Components of vectors $\hat{\mathbf{L}}$ and $\hat{\mathbf{A}}$ can be combined to yield generators of the so(4) symmetry [2] of Hamiltonian (39).

Let us show that conservation laws (40) can be derived directly from equation (3) using only the knowledge of exact eigenstates of Hamiltonian (39). The conservation of angular momentum is not 
specific to $1 / r$ potential. We therefore assume that we know that the angular momentum is conserved, but do not know any other conservation laws of Hamiltonian (39).

According to (3) the conservation laws are of the form

$$
\hat{X}=\sum_{n} \hat{P}_{n} \hat{Y} \hat{P}_{n}
$$

where $\hat{P}_{n}$ projects out the eigenspace of (39) with eigenvalue $E_{n}=-1 / 2 n^{2}$. We take a straightforward approach and try the simplest choices for $\hat{Y}$.

First, we look for a conserved scalar and try $\hat{Y}_{1}=\hat{\mathbf{r}}^{2}$ and $\hat{Y}_{2}=\hat{\mathbf{p}}^{2}$. This choice as well as any other powers of $|\hat{\mathbf{p}}|$ and $|\hat{\mathbf{r}}|$ result only in trivial commuting operators - combinations of $\hat{H}$ and $\hat{\mathbf{L}}^{2}$. For example,

$$
\hat{X}_{1}=\sum_{n} \hat{P}_{n} \hat{\mathbf{r}}^{2} \hat{P}_{n}=\frac{1}{4 \hat{H}}\left(\frac{5}{2 \hat{H}}+\hat{\mathbf{L}}^{2}-1\right)
$$

Next, we search for a conserved vector and try $\hat{Y}=\hat{\mathbf{r}}$. To evaluate

$$
\hat{\mathbf{B}}=\sum_{n} \hat{P}_{n} \hat{\mathbf{r}} \hat{P}_{n}
$$

explicitly it is advantageous to use parabolic coordinates. The eigenstates of discrete spectrum are specified by three integers $\left|n_{1}, n_{2}, m\right\rangle$, where $m$ is the z-projection of the angular momentum and the principle quantum number is $n=n_{1}+n_{2}+|m|+1$ (see e.g. [1]). Evaluating the matrix elements of $B_{z}$ in this basis, we find that only diagonal matrix elements are nonzero and

$$
\hat{B}_{z}\left|n_{1}, n_{2}, m\right\rangle=\frac{3 n}{2}\left(n_{1}-n_{2}\right)\left|n_{1}, n_{2}, m\right\rangle
$$

To express $\hat{B}_{z}$ through components of $\hat{\mathbf{r}}$ and $\hat{\mathbf{p}}$ we have to identify operators that have eigenvalues $n, n_{1}$, and $n_{2}$. All we need to do is to follow backwards the derivation of eigenstates in parabolic coordinates. Substituting these operators instead of $n, n_{1}$, and $n_{2}$ into equation (43), we find

$$
\hat{B}_{z}=-\frac{3 \hat{A}_{z}}{4 \hat{H}}
$$

Since the choice of z-axis is arbitrary, we conclude that

$$
\hat{\mathbf{B}}=-\frac{\hat{\mathbf{A}}}{4 \hat{H}}
$$

with $\hat{\mathbf{A}}$ given by equation (40).

Thus, the substitution of $\hat{Y}=\hat{\mathbf{r}}$ into equation (3) produces the Runge-Lenz vector - the nontrivial conservation law responsible for the "accidental" degeneracy in Hydrogen atom. One can check that the alternative choice $\hat{Y}=\hat{\mathbf{p}}$ yields the same result. 


\section{Conclusion}

We have derived a complete set of symmetry generators of Hamiltonian (1) at $x=1$ and established that the symmetry group of the Hamiltonian is $S U(2)$. The degenerate subspace transforms under a $(2 K+1)$-dimensional representation of this $S U(2)$, nondegenerate states being singlets. For $K \gg 1$ the degenerate states correspond to $\hat{S}_{\mathbf{n}}=0$ where $\mathbf{n}$ is a unit vector along $\mathbf{z}+\hat{\mathbf{K}} / K$ and the symmetry operators have a meaning of rotations of $\hat{\mathbf{K}}$ for these states. We outlined a general approach for finding symmetries that can be used when the eigenstates and eigenvalues of the Hamiltonian are known. The formal starting point in this approach is the general expression for conservation laws (3). We have seen that for both Hamiltonian (1) and the Hydrogen atom simplest choices for $\hat{Y}$ produce all nontrivial conservation laws.

We do not have a satisfactory explanation of why multiple degeneracies at nonzero value of $x$ occur only for $S=1$ and not for other integer values of $S$. If this question is to be answered on the symmetry grounds, further insight into the nature and simple manifestations of symmetries derived in this paper is needed. In particular, the analysis of subleading corrections to large $K$ limit may be an interesting avenue to pursue.

\section{Appendix: Commuting Operators}

Here we prove equation (3) for Hamiltonian (1). The proof can be readily generalized to any Hermitian operator whose eigenstates span the Hilbert space.

In the energy representation Hamiltonian (1) can be written as

$$
\hat{H}=\left[\begin{array}{cc}
-\frac{1}{2} \mathcal{E} & 0 \\
0 & \mathcal{D}
\end{array}\right]
$$

where $\mathcal{E}$ is a $(2 K+1) \times(2 K+1)$ identity matrix and $\mathcal{D}$ is a diagonal matrix with no two diagonal elements equal to each other or to $-1 / 2$. The block $-1 / 2 \mathcal{E}$ corresponds to the degenerate subspace, $\mathcal{D}$ to the remaining levels. Since any matrix commutes with an identity matrix and only diagonal

matrices commute with $\mathcal{D}$, an operator $\hat{X}$ commutes with the Hamiltonian if and only if it is of the form

$$
\hat{X}=\left[\begin{array}{cc}
\mathcal{A} & 0 \\
0 & \mathcal{D}^{\prime}
\end{array}\right]=\left[\begin{array}{ll}
\mathcal{A} & 0 \\
0 & 0
\end{array}\right]+\left[\begin{array}{cc}
0 & 0 \\
0 & \mathcal{D}^{\prime}
\end{array}\right]
$$

where $\mathcal{A}$ is an arbitrary $(2 K+1) \times(2 K+1)$ matrix and $\mathcal{D}^{\prime}$ is a diagonal matrix of an appropriate size. Using the projection operator $\hat{P}$, we can rewrite (A.2) as

$$
\hat{X}=\hat{P} \hat{Y} \hat{P}+\hat{\Lambda}
$$


where $\hat{Y}$ is an arbitrary operator and $\hat{\Lambda}$ represents the second operator on the left hand side of equation (A.2). The action of the operator $\hat{\Lambda}$ on the degenerate subspace is trivial. Namely,

$$
\hat{\Lambda}|D m\rangle=0
$$

Thus, with no loss of generality the search of commuting operators can be restricted to operators of the form

$$
\hat{X}=\hat{P} \hat{Y} \hat{P}
$$

\section{Acknowledgment}

We are grateful to Natalia Saulina for helpful discussions. B. L. A. also acknowledges travelling support of the EPSRC under the grant GR/R33311.

\section{References}

[1] Landau L. D. and Lifshitz E. M. 1958 Quantum Mechanics (London)

[2] Pauli W., 1926 Z. Phys 36336

Fock V. A., 1935 Zs. f. Physik 98145

[3] C. J. Erickson, D. Levron, W. Happer, S. Kadlecek, B. Chann, L. W. Anderson, T. G. Walker, Phys. Rev. Lett. 20 v. 85 p. 4237 (2000).

[4] C. M. Bai, M. Ge, K. Xue, cond-mat/0105610

[5] M. H. Hebb, Phys. Rev. 49, p. 610 (1936); C. H. Townes and A. L. Schawlow, Microwave Spectroscopy, p. 182, Dover Publications, New York (1975)

[6] W. Happer, unpublished manuscript.

[7] Haymaker R. W. and Rau A. R. R. 1986 Amer. J. Phys. 54928. 